\title{
Entre erros e acertos: promovendo a segurança do paciente a partir da insegurança noticiada
}

Elaine Cristina Novatzki Forte ${ }^{1}$, Denise Elvira Pires de Pires ${ }^{2}$, Maria Manuela Ferreira Pereira da Silva Martins ${ }^{3}$, Maria Itayra Coelho de Souza Padilha ${ }^{4}$, Dulcinéia Ghizoni Schneider ${ }^{5}$, Letícia de Lima Trindade ${ }^{6}$

\section{RESUMO}

Estudo qualitativo, documental, interpretativo, com coleta de dados realizada em jornais de dois países, com o objetivo de interpretar o modo como as notícias sobre erros de enfermagem na mídia podem contribuir para a compreensão das questões relacionadas à segurança do paciente. Realizada análise hermenêutica com base no Processo de Trabalho e na Teoria do Agir Comunicativo, com auxílio do software Atlas.ti. Resultaram três categorias temáticas que permitiram compreender os problemas relacionados ao financiamento da saúde, às oportunidades de proporcionar a compreensão do problema à sociedade e às possibilidades de representar e fortalecer a identidade profissional. Conclui-se que os meios de comunicação podem ser importantes aliados da enfermagem na busca por melhores condições de trabalho e para dar visibilidade à problemática em si e ao subfinanciamento da saúde, pois as notícias permitem aproximações e distanciamentos, se inseridas no contexto histórico, político e social dos envolvidos.

Descritores: Segurança do Paciente; Meios de Comunicação; Enfermagem; Notícias; Gerenciamento de Riscos.

\footnotetext{
${ }^{1}$ Enfermeira, Doutora em Enfermagem - Educação e Trabalho em Saúde e Enfermagem. Professor Substituto da Universidade Federal de Santa Catarina. Florianópolis, SC, Brasil. E-mail: naneforte@yahoo.com.br.

${ }^{2}$ Enfermeira, Doutora em Ciências Sociais. Professor Titular da Universidade Federal de Santa Catarina. Florianópolis, SC, Brasil. E-mail: piresdp@yahoo.com.

${ }^{3}$ Enfermeira, Doutora em Ciências de Enfermagem. Docente da Escola Superior de Enfermagem da Universidade do Porto. Porto, Portugal. E-mail: mmartins@esenf.pt.

${ }^{4}$ Enfermeira, Doutora em Enfermagem. Professora Titular da Universidade Federal de Santa Catarina. Florianópolis, SC, Brasil. E-mail: itayra.padilha@ufsc.br.

${ }^{5}$ Enfermeira, Doutora em Enfermagem. Professora Adjunta da Universidade Federal de Santa Catarina. Florianópolis, SC, Brasil. E-mail: dulcineia.schneider@ufsc.br.

${ }^{6}$ Enfermeira, Doutora em Enfermagem. Professora Adjunta da Universidade do Estado de Santa Catarina. E-mail: letrindade@ hotmail.com.
}

\section{Como citar esse artigo:}

Forte ECN, Pires DEP, Martins MMFPS, Padilha MICS, Schneider DG, Trindade LL. Entre erros e acertos: promovendo a segurança do paciente a partir da insegurança noticiada. Rev. Eletr. Enf. [Internet]. 2018 [acesso em: ];20:v20a50. Disponível em: https://doi.org/10.5216/ree.v20.52539. 


\section{INTRODUÇÃO}

Com a expansão dos meios de comunicação em massa, as informações passaram a ser fornecidas numa velocidade difícil de ser acompanhada. E nesse cenário, a saúde ocupa os conteúdos midiáticos com assuntos relacionados à qualidade da assistência em saúde, em especial no que se refere à superlotação dos serviços de emergência, à carência de profissionais de saúde, ao financiamento dos serviços e aos erros decorrentes dos cuidados $^{(1)}$. Os erros assistenciais e eventos adversos em saúde têm se tornado alvo de estudos em muitos países, principalmente pela alta incidência e pelas consequências desses erros, e esse problema não é localizado, nem tampouco de fácil resolução, por isso requer discussões contínuas com diferentes abordagens.

Para isso, existe um forte movimento mundial sinalizando mudanças importantes no contexto dos cuidados de saúde com a finalidade de garantir ambientes mais seguros e de qualidade. Essas mudanças têm sido implementadas com base em esforços múltiplos provenientes de estudos que foram essenciais para dar respaldo científico a essas mudanças. As questões de segurança do paciente e da qualidade da assistência em saúde, sendo uma demanda urgente deste século, fomentaram a criação do projeto "Aliança Mundial para a Segurança do Paciente" da Organização Mundial da Saúde (OMS), com o intuito de estimular melhorias por meio de campanhas e programas por todo o mundo(2).

Nessa vertente, cada país configurou o seu próprio plano para melhorar a segurança dos cuidados e ir ao encontro das metas estabelecidas mundialmente. No Brasil, o Programa Nacional de Segurança do Paciente (PNSP) foi criado em 2013 para diminuir a incidência de erros (ou eventos adversos, na terminologia utilizada pela OMS) e mais tarde institui-se a obrigatoriedade de implantar o Núcleo de Segurança do Paciente (NSP), para colocar em prática os planos de segurança e notificar os eventos adversos ${ }^{(2)}$.

Em Portugal, com base nos determinantes para a qualidade nos serviços de saúde estipulados pela DireçãoGeral da Saúde, a Ordem dos Enfermeiros (OE) revisou e atualizou os Padrões de Qualidade dos Cuidados de Enfermagem, um documento oficial que visa estimular cuidados de enfermagem seguros e de qualidade em todo o território português ${ }^{(3-4)}$.

Embora as ações realizadas em prol de cuidados mais seguros em todo mundo tenham estimulado e modificado consideravelmente as práticas de saúde no mundo - como verificado em hospitais da Holanda que conseguiram reduzir $45 \%$ dos eventos adversos evitáveis num período de quatro anos analisados em 16.000 internações ${ }^{(5)}$ - é comum ainda casos de erros nos cuidados em saúde, em geral, serem noticiados, seja na mídia jornalística escrita e televisão, seja nas redes sociais e outras formas de comunicação.

Surge, então, a necessidade de expandir o olhar para essa problemática utilizando uma forma que busque identificar nos textos midiáticos maneiras de compreender a complexidade do erro de enfermagem divulgado. Essa compreensão ultrapassa a maneira clássica causa-efeito de entender determinados fenômenos, e busca na interpretação revelar conteúdos, até então, imersos na linguagem. Estudos anteriores mostraram o predomínio de erros de medicamentos em notícias de jornais e classificaram como gravíssimos a maioria dos achados, confirmando a necessidade de melhorar os sistemas de medicação nas instituições de saúde ${ }^{(1,6)}$.

Este estudo se justifica pela temática da segurança do paciente que deve ser incansável e abrangente, e pela maneira que a informação é repassada à sociedade, para compatibilizar com a complexidade que envolve esses incidentes, considerando que os meios de comunicação se constituem ferramentas eficazes para a 
divulgação, esclarecimento, problematização e compreensão de determinados fenômenos. Ademais, constatouse a incipiência de estudos dessa natureza, tendo em vista que a maioria dos estudos acerca dessa temática, busca identificar fatores de risco associados aos erros e suas complicações ${ }^{(7)}$. Assim, tem como propósito interpretar de que modo as notícias sobre erros de enfermagem podem contribuir para a compreensão das questões relacionadas à segurança do paciente.

\section{MÉTODO}

Este estudo documental, retrospectivo, de abordagem qualitativa e interpretativa, utilizou como fonte de dados notícias veiculadas nos jornais de grande circulação no Brasil e em Portugal, no período janeiro de 2012 até o mês de dezembro de 2016. A coleta de dados por meio de clipagem ou assinatura temporária dos jornais ocorreu entre outubro de 2015 e dezembro de 2016 e levantou todas as notícias referentes a erros de enfermagem divulgados na mídia.

Compôs o corpus da pesquisa 112 notícias divulgadas que atenderam ao critério de inclusão: textos completos noticiados que tratam de erro decorrente da assistência de enfermagem. Destas, 18 notícias foram publicadas em Portugal e 94 no Brasil. Foram excluídas notícias que tratavam de erros de outros profissionais, relacionadas a outras condutas consideradas erradas, como furtos e assédios e textos em que não foi explicitada a participação da enfermagem, tendo em vista que essa informação se constitui condição sine qua non para este estudo.

O dados coletados foram armazenados no software Atlas.ti e a análise dos textos utilizou como referencial a teorização sobre processo de trabalho de Karl Marx e a teoria do agir comunicativo de Jürgen Habermas, com o objetivo de compreender, por meio da comunicação, as formas manifestas do trabalho de enfermagem que ocultam problemas de ordem social, dentro de um contexto histórico. Para tanto, recorreu-se à hermenêutica, através dos passos sugeridos por Paul Ricoeur para chegar ao objetivo proposto, que se constituem na leitura inicial do texto (criadas as primeiras notas de registro), na busca de sentidos, e na apropriação (quando a mensagem é revelada). Esse processo permite mostrar um sentido explícito e esconder outro que só pode ser compreendido através do primeiro(8).

Nomeadamente à ética, os dados coletados estão à disposição da sociedade por meio eletrônico, e não envolve diretamente seres humanos, o que dispensa análise de comitês de ética em pesquisa. Contudo, os profissionais de enfermagem, pacientes, familiares, jornais e jornalistas, não foram identificados. As notícias foram identificadas por códigos compostos pela letra J, com a letra inicial do país (B - Brasil e P - Portugal), acrescido das letras que identificam a região e um número de ordem.

Ao final, três categorias analíticas principais foram construídas, e as informações dos erros foram associadas com possíveis unidades de interpretação do conteúdo a fim de propiciar formas de entendimento que possibilitem a compreensão da problemática, sem inferir julgamentos. Caracterizou-se num movimento dialético no qual o explícito e o implícito nos textos foram articulados de maneira que as categorias formuladas sinalizam a relevância para essa forma de olhar a segurança. 


\section{RESULTADOS E DISCUSSÃO}

Da análise interpretativa dos resultados, resultaram três temas principais, cada qual relacionado às informações codificadas nos textos em conjunto com possíveis núcleos de sentido que possam contribuir, de alguma forma, para compreender a magnitude do problema e a reflexão acerca das possibilidades que a exposição midiática pode resultar, visto a complexidade desses incidentes noticiados.

\section{O dinheiro como fator implícito de risco}

Desde sempre o fator financiamento dos serviços de saúde é um problema em diversos países. O dinheiro, de forma ambígua, é o problema e a solução para a maioria das questões de saúde, haja vista que, quanto maior o investimento nas ações de saúde, em todos os âmbitos da assistência à população melhores os resultados de saúde, e a sua ausência/insuficiência tem um impacto direto nos resultados negativos.

Os investimentos no setor saúde, assim como a gestão dos recursos, têm sido motivo de discussões justamente pelos déficits orçamentários que os países têm sofrido constantemente, em especial, por não colocarem a saúde como uma prioridade da atuação política e administrativa ${ }^{(9-10)}$. A saúde é vista pela população como uma prioridade na maioria dos países, com exceção de alguns casos em que outras questões relacionadas à sobrevivência mínima, como o emprego e a remuneração, são vistos com maior relevância(11).

Nessa acepção, não há como desvincular o problema de financiamento da saúde com as questões de segurança do paciente e a qualidade dos cuidados, existe sim uma forte influência do incentivo financeiro para o gerenciamento de riscos e acidentes. Logo, a partir do que foi noticiado acerca dos erros de enfermagem foi concebido um quadro com a identificação das informações explícitas que possibilitaram a associação com os problemas de ordem orçamentária.

Quadro 1: Tipos de erros, consequências, causas atribuídas e os fatores de risco relativos ao financiamento do setor saúde.

\begin{tabular}{|c|c|c|c|c|}
\hline Tipo de erro & Consequência & Causa atribuída & Fator de risco & $\begin{array}{c}\text { Fator } \\
\text { implícito }\end{array}$ \\
\hline \multicolumn{3}{|c|}{ Dados explícitos nas notícias analisadas } & \multicolumn{2}{|c|}{ Dados relacionados com os erros noticiados } \\
\hline $\begin{array}{c}\text { Medicação - troca } \\
\text { de vias }\end{array}$ & $\begin{array}{l}\text { Complicações no quadro } \\
\text { de saúde, aumento do } \\
\text { tempo de internação }\end{array}$ & \multirow{3}{*}{$\begin{array}{c}\text { Sobrecarga de } \\
\text { trabalho, precariedade } \\
\text { de equipamentos }\end{array}$} & \multirow{3}{*}{$\begin{array}{l}\text { Déficit de profissionais, } \\
\text { rotatividade de profissionais, } \\
\text { problemas estruturais }\end{array}$} & \multirow{6}{*}{ 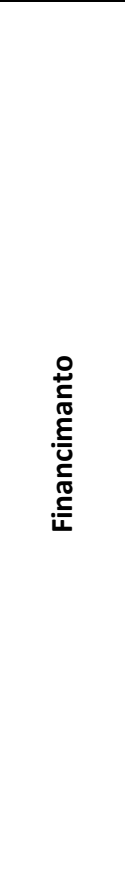 } \\
\hline Infecções & $\begin{array}{l}\text { Morte, agravamento no } \\
\text { quadro de saúde, aumento } \\
\text { do tempo de internação }\end{array}$ & & & \\
\hline Quedas & $\begin{array}{l}\text { Fratura, cirurgia, aumento } \\
\text { do tempo de internação }\end{array}$ & & & \\
\hline $\begin{array}{l}\text { Medicação - troca } \\
\text { de substâncias }\end{array}$ & $\begin{array}{l}\text { Morte, sequelas, } \\
\text { internação prolongada }\end{array}$ & $\begin{array}{l}\text { Semelhança de rótulos } \\
\text { e embalagens, } \\
\text { problemas de } \\
\text { armazenamento }\end{array}$ & $\begin{array}{c}\text { Problemas relacionados com a } \\
\text { compra de materiais e insumos, } \\
\text { problemas estruturais }\end{array}$ & \\
\hline $\begin{array}{l}\text { Esquecimento de } \\
\text { material (cirúrgico } \\
\text { ou ambulatorial) }\end{array}$ & $\begin{array}{l}\text { Cirurgia, aumento do } \\
\text { tempo de internação, } \\
\text { morte, complicações no } \\
\text { quadro de saúde }\end{array}$ & $\begin{array}{l}\text { Distração, sobrecarga } \\
\text { de trabalho, } \\
\text { negligência profissional }\end{array}$ & $\begin{array}{c}\text { Déficit de profissionais, } \\
\text { rotatividades de profissionais, } \\
\text { falta de capacitação/educação } \\
\text { permanente }\end{array}$ & \\
\hline $\begin{array}{l}\text { Classificação de } \\
\text { risco }\end{array}$ & $\begin{array}{c}\text { Morte e complicações no } \\
\text { quadro de saúde }\end{array}$ & Sobrecarga de trabalho & $\begin{array}{l}\text { Déficit de profissionais, } \\
\text { rotatividade de profissionais, falta } \\
\text { de capacitação/educação } \\
\text { permanente }\end{array}$ & \\
\hline
\end{tabular}


No Brasil, o gasto público em saúde tem ficado um pouco acima de $40 \%$ do total dos gastos em saúde no país e não deve melhorar substancialmente nos próximos anos ${ }^{(12-13)}$. Esse pessimismo é reforçado, em especial, devido à aprovação da Emenda Constitucional no 95/2016 que instituiu um novo regime fiscal e limitou os gastos públicos nos próximos 20 anos. Portanto, contrariando a marcha que caminham os países desenvolvidos, em que o investimento público, da média dos países da Organização para Cooperação de Desenvolvimento Econômico (OCDE), supera os $70 \%$ (em relação ao total de gastos em saúde) ${ }^{(12)}$, esse patamar deve continuar ou crescer, na medida em que cresce o debate sobre o direito universal à saúde.

Em Portugal, desde a crise financeira instalada em 2010, quando houve um corte severo nos gastos e aumento significativo de impostos, os problemas na saúde emergiram ${ }^{(14)}$. Face à necessidade urgente de contenção de despesas, a área que mais sofreu as consequências da crise, em curto prazo, foi a da saúde. Não é à toa que se assiste quase que mensalmente, as ameaças ou mesmo a concretização das greves dos enfermeiros no país. São milhares de horas devidas aos profissionais que, já por contrato, não recebem um salário de acordo com o esperado para a profissão e nem ao menos, têm suas especialidades reconhecidas ${ }^{(15)}$.

A grande questão levantada aqui é, quanto menor o investimento em ações que aumentem a segurança dos pacientes nos ambientes de trabalho, maior será o gasto com as consequências dos erros e acidentes gerados por esses ambientes, ditos inseguros. $O$ aumento do tempo de internação, as internações em leitos de Unidade de Terapia Intensiva (UTI), a realização de novas cirurgias (não previstas) e outras formas de tratamento, recuperação e reabilitação, podem gerar despesas exorbitantes não previstas no orçamento das instituições, sendo que esses custos podem aumentar mais de $200 \%$ se comparado aos gastos previstos com pacientes que não sofreram esses incidentes ${ }^{(16)}$.

A questão dos gastos também se estende aos casos que acabam por se tornar alvo de processos litigiosos que podem gerar gastos elevados, principalmente com as indenizações previstas em legislação específica de cada país. No caso do Brasil, a Constituição Federal, o Código de Defesa do Consumidor (CDC) e o Código de Processo Civil sustentam os direitos dos consumidores da assistência em saúde na busca por indenizações por dano físico, material e moral.

Constitui-se, portanto, um ciclo resultado-consequência-resultado, em que a falta de investimento na força de trabalho e na estrutura das instituições com vistas a favorecer a segurança, geram consequências negativas para a saúde da população, que por sua vez precisa de mais assistência além da necessária, impactando no orçamento. E assim, com o orçamento comprometido, torna-se cada vez mais difícil investir na força de trabalho e na estrutura para melhor gerenciar os riscos decorrentes dos cuidados de saúde.

\section{O erro como despertar - expor para conscientizar}

A exposição midiática é uma forma eficaz de levantar questões que parecem imersas na complexidade dos serviços de saúde. Nesse sentido, a mídia possui o poder de influenciar opiniões, mediando contextos e regras da sociabilidade contemporânea, constituindo-se num território no qual é regulada a existência das coletividades na atualidade $^{(17)}$.

Os comunicados sobre saúde na mídia têm em consideração três aspectos: o contexto da produção de notícias, a lógica capitalista dos diferentes meios de comunicação e o público ao qual a informação é destinada. 
Assim, a saúde é um tema recorrente e de grande repercussão por atender as questões de cunho comercial (audiência e lucro) e para a conquista do público(18).

A maior visibilidade midiática para os erros decorrentes da assistência em saúde parte de casos como o da jornalista do Boston Globe, Betsy Lehman, que morreu depois de receber altas doses de ciclofosfamida durante quatro dias consecutivos. Esse episódio fez com que ações no âmbito da segurança na administração de medicamentos fossem implementadas, e a equipe de profissionais que assistiu a jornalista não sofreu qualquer penalidade, pois houve a conscientização para o problema de forma a evitar futuros incidentes. O fato em si desencadeou na criação do "Betsy Lehman Center for Patient Safety and Medical Error Reduction" que visa à diminuição de erros de medicação na assistência hospitalar ${ }^{(19)}$.

Nesse sentido, os erros noticiados podem servir de impulso para ações de segurança, tanto por parte dos profissionais, quanto pelas instituições de saúde, assim como pelos pacientes, que tendem a se tornar mais vigilantes com os seus cuidados. Nos casos noticiados, evidencia-se que as denúncias de erros podem servir para repensar as práticas de enfermagem, pois há um temor nos profissionais de que o erro com graves consequências possa se tornar uma realidade. O medo também é um fator protetivo e faz as pessoas (re)pensarem a sua prática diária, saindo do tecnicismo automático.

Em alguns casos, o próprio familiar da vítima reconhece o caráter educativo que têm a visibilidade do erro e as consequências para os profissionais. Ademais, as instituições de saúde passam a rever seus processos assistenciais e a sinalizar equipamentos/medicamentos que representam maior perigo.

Logo depois da troca dos medicamentos, os pais do menino procuraram a Polícia Civil e fizeram um BO sobre o caso. "Não fiz isso para prejudicar ninguém, mas para servir de alerta para outras pessoas" (JBPR11).

o Hospital de Braga assumiu, logo na altura, "erro humano" na programação do equipamento e anunciou que, atendendo à gravidade do sucedido, a comissão executiva desencadeou "de imediato" um processo de identificação de medidas suplementares para obstar a ocorrência de novos erros (JPMI3).

A forma como é noticiado pode induzir à ideologias que colaboram para a segurança nos serviços de saúde, não a ideologia na forma de poder e dominação, mas sim para a organização da sociedade, pois servem para direcionar as pessoas, intelectual e moralmente, nas condutas e pensamentos diários. Por isso, as instituições de saúde devem evitar as ações punitivas, assim como os meios de comunicação devem evitar transmiti-las de maneira incisiva e parcial, para que se estimule cada vez mais uma cultura de segurança universal, sem culpados, sem inocentes, somente pensando no melhor de si, para si e para o outro.

\section{Lutas constantes para glórias futuras - representação e identidade profissional}

As exposições da enfermagem na mídia, a depender da maneira como são publicadas as notícias, podem se constituir num veículo de representação e fortalecimento da identidade profissional. Mesmo que em situações muito adversas, como são os casos de erros assistenciais, essa se configura numa oportunidade ímpar de mostrar para a sociedade aspectos da profissão pouco reconhecidos ou, até mesmo, desconhecidos.

As oportunidades concedidas para a representação profissional foram identificadas em 23 notícias, com especial atenção para as notas expedidas pelos órgãos de representação profissional. As notas se referem a informações que visam proteger os profissionais acusados de erro, especialmente quando estas informações 
mostram a participação constante na fiscalização dos serviços de saúde e na maneira como os processos administrativos ou judiciais estão sendo conduzidos. As notas também provêm do judiciário que faz reconhecer as situações problemáticas no trabalho saúde, como visto na fala reproduzida de uma desembargadora em sentença:

Na opinião da magistrada, era inevitável que em algum momento os problemas enfrentados na ponta da saúde pública resultassem em ações na Justiça. "Todos os dias, o que vemos são hospitais em situações precárias de atendimento, com equipes desfalcadas, falta de medicamentos, insumos e equipamentos. Todas essas situações provocam uma pressão no profissional, que trabalha cada vez mais sobrecarregado e sujeito a cometer erros", afirma. Com alta demanda e poucas equipes, pacientes e médicos ficam na mesma situação (JBMG10).

Há uma dicotomia na forma como a mídia tem trabalhado determinadas situações que giram em torno do benefício da circulação de informações de forma irrestrita e veloz, e o direito de preservar a intimidade e a privacidade das pessoas, ou seja, são dois aspectos tanto jurídicos como sociais: a proteção da privacidade (cada vez mais vulnerável) e a liberdade de expressão da imprensa ${ }^{(20)}$.

Dessa forma, ao propiciar um espaço para que as entidades representativas da enfermagem esclareçam situações e, mesmo questionem algumas das informações apresentadas, novas formas de entendimento podem ser divulgadas, protegendo a imagem da profissão. O contexto atual da enfermagem nos dois países pesquisados é caracterizado por lutas constantes por melhores condições de trabalho. No Brasil existem dois projetos de lei que tentam há anos regulamentar a jornada de trabalho e a remuneração da enfermagem, pois as condições de trabalho são, de fato, um requisito para práticas de enfermagem mais seguras ${ }^{(21)}$. Entretanto, essa é uma questão de difícil resolução que tem se arrastado por décadas, principalmente, porque envolve recursos financeiros, e quando se trata da saúde, essa não é uma prioridade na agenda política do país.

Em Portugal, essa situação não é muito diferente. As greves são constantes e todos os anos as demandas são as mesmas: jornada de trabalho, salário, horas extras, enfermeiros especialistas sem o devido reconhecimento e déficit de profissionais. Nesse quesito, a Ordem dos Enfermeiros mantém-se apoiante das greves e das reivindicações, e para isso utiliza seus próprios informativos e notas na imprensa ${ }^{(22)}$.

Desse mesmo modo, os conselhos e os sindicatos brasileiros tentam dar visibilidade às lutas da enfermagem, tendo em vista a valorização da profissão e o resgate da identidade profissional, no que "diz respeito à percepção externa (positiva ou negativa) que a sociedade e/ou indivíduo podem ter de uma pessoa, instituição ou organização"(23). A imagem da enfermagem não deve estar associada ao resultado negativo de seu trabalho. Quando incidentes de erros ocorrem, estes devem ser compreendidos na sua totalidade, evitando que a sociedade transponha valores negativos a toda profissão com base em fatos isolados, e muitas vezes, pouco esclarecidos.

A exposição midiática pode contribuir para que a sociedade compreenda melhor a complexidade do problema e ainda, pode se constituir em um espaço de apoio para a profissão, para suas lutas e conquistas futuras, que são para o bem de todos.

\section{CONSIDERAÇÕES FINAIS}

Interpretar é sempre uma tarefa árdua e requer olhar atento do pesquisador para o que não se mostra naquela que é a fonte dos dados. Mas é possível e pode explanar outras formas de olhar para o mesmo fenômeno, 
de modo a expandir a investigação para além do que a priori, foi proposto. Este estudo permite afirmar que os meios de comunicação em massa, como os jornais, podem ser importantes aliados da enfermagem na busca por melhores condições de trabalho, contribuindo para dar visibilidade à problemática em si e ao subfinanciamento da saúde.

Embora as notícias não sejam totalmente claras, os textos sempre permitem aproximações e distanciamentos em relação às informações, inserindo-as no contexto histórico, político e social dos envolvidos, para aprofundar determinados aspectos relevantes para a compreensão.

No que tange à relevância social, este estudo aponta para os cuidados que os jornalistas devem ter na veiculação de informações ao retratar esses incidentes, que estão atrelados à clareza das informações, às questões do âmbito da ética e da política, à parcialidade das informações de pacientes/familiares, e ao real objetivo para a sociedade. Do ponto de vista científico, esta maneira de investigar o fenômeno erro de enfermagem contribuiu para a compreensão da diversidade das nuances que evolve o trabalho da enfermagem e da sua importância social, haja vista as muitas adversidades para um trabalho com qualidade segurança.

\section{REFERÊNCIAS}

1. Volpe CRG, Aguiar LB, Pinho DL, Stival MM, Funghetto SS, Lima LR. Erros de medicação divulgados na mídia: estratégias de gestão do risco. Revista de Administração Hospitalar e Inovação em Saúde [Internet]. 2016 [cited 2018 Dec 31];13(2):97-110. Available from: https://doi.org/10.21450/rahis.v13i2.3499.

2. Andrade LEL, Lopes JM, Souza Filho MCM, Vieira Júnior RF, Farias LPC, Santos CCM, et al. Cultura de segurança do paciente em três hospitais brasileiros com diferentes tipos de gestão. Cien Saude Colet [Internet]. 2018 [cited 2018 Dec 31]; 23(1):161-72. Available from: https://doi.org/10.1590/1413-81232018231.24392015.

3. Ribeiro O, Martins MMFPS, Tronchin DMR. Qualidade dos cuidados de enfermagem: um estudo em hospitais portugueses. Revista de Enfermagem Referência [Internet]. 2017 [cited 2018 Dec 31]; IV(14):89-100. Available from:

https://doi.org/10.12707/RIV16086.

4. Martins MMFPS, Gonçalves MNC, Ribeiro OMPL, Tronchin DM. Qualidade dos cuidados de enfermagem: construção e validação de um instrumento. Rev Bras Enferm [Internet]. 2016 [cited 2018 Dec 31];69(5):864-70. Available from:

https://doi.org/10.1590/0034-7167-2015-0151.

5. Baines R, Langelaan M, Bruijne M, Spreeuwenberg $P$, Wagner C. How effective are patient safety initiatives? A retrospective patient record review study of changes to patient safety over time. BMJ Qual Saf [Internet]. 2015 [cited 2018 Dec 31];24(9):561-71. Available from: https://doi.org/10.1136/bmjqs-2014-003702.

6. Fontana RT, Wolf J, Rodrigues FCP, Castro LM. Análise documental da mídia escrita sobre eventos adversos ocorridos na prática da enfermagem. Revista de Enfermagem UFPE Online [Internet]. 2015 [cited 2018 Dec 31];9(Supl 4):8103-10. Available from: https://periodicos.ufpe.br/revistas/revistaenfermagem/article/view/10565.

7. Forte ECN, Pires DEP, Padilha MI, Martins MMFPS. Nursing errors: a study of the current literature. Texto contexto - enferm [Internet]. 2017 [cited 2018 Dec 31];26(2):e01400016. Available from: https://doi.org/10.1590/0104-07072017001400016. 8. Forte ECN, Pires DEP, Trigo SVVP, Martins MMFPS. A hermenêutica e o software Atlas.ti: união promissora. Texto contexto enferm [Internet]. 2017 [cited 2018 Dec 31];26(4):e0350017. Available from: https://doi.org/10.1590/0104-07072017000350017. 9. Gomes FBC. Impasses no financiamento da saúde no Brasil: da constituinte à regulamentação da emenda 29/00. Saúde em Debate [Internet]. 2014 [cited 2018 Dec 31];38(100):6-17. Available from: https://doi.org/10.5935/0103-104.20140001.

10. Vieira FS, Benevides RPS. Os impactos do novo regime fiscal para o financiamento do Sistema Único de Saúde e para a efetivação do direito à saúde no Brasil. Nota Técnica. № 28 [Internet]. Brasília: IPEA, 2016 [cited 2018 Dec 31]. Available from: http://repositorio.ipea.gov.br/bitstream/11058/7270/1/NT n28 Disoc.pdf.

11. Lorenzetti J, Lanzoni GMM, Assuiti LFC, Pires DEP, Ramos FRS. Health management in Brazil: dialogue with public and private managers. Texto contexto - enferm [Internet]. 2014 [cited 2018 Dec 31];23(2):417-25. Available from:

https://doi.org/10.1590/0104-07072014000290013.

12. Organisation for Economic Co-operation and Development. Health at a Glance 2015 [Internet]. Paris: OECD; 2015 [cited 2018 Dec 31]. Available from: https://doi.org/10.1787/health glance-2015-en. 
13. Afonso JR, Castro KP. A crise (do financiamento) da saúde. Conjuntura Econômica [Internet]. 2016 [cited 2018 Dec 31];70(5):224. Available from: http://bibliotecadigital.fgv.br/ojs/index.php/rce/article/view/64663/62544.

14. Costa $\mathrm{H}$. Le syndicalisme portugais et l'austérité : entre la force des protestations et la fragilité des alliances. Relations industrielles [Internet]. 2015 [cited 2018 Dec 31];70(2):262-84. Available from: https://doi.org/10.7202/1031464ar.

15. Costa HA, Dias H, Soeiro J. As greves e a austeridade em Portugal: olhares, expressões e recomposições. Revista Crítica de Ciências Sociais [Internet]. 2014 [cited 2018 Dec 31];103:173-202. Available from: https://doi.org/10.4000/rccs.5584.

16. Porto S, Martins M, Mendes W, Travassos C. A magnitude financeira dos eventos adversos em hospitais no Brasil. Revista Portuguesa de Saúde Pública [Internet]. 2010 [cited 2018 Dec 31];(10):74-80. Available from: http://www.elsevier.es/en-revistarevista-portuguesa-saude-publica-323-articulo-a-magnitude-financeira-dos-eventos-X0870902510898606.

17. Rocha RL. Os negócios da mídia e a comunicação da saúde. Cad Saude Publica [Internet]. 2016 [cited 2018 Dec 31];32(2):e00000616. Available from: https://doi.org/10.1590/0102-311X00000616.

18. Martinez M, Pessoni A, Silva MCC, Ribeiro V. Assessoria de imprensa, narrativas midiáticas e saúde: simbiose de fontes, jornalistas, leitores, personagens e afetos. Intexto [Internet]. 2017 [cited 2018 Dec 31];(38):197-224. Available from: https://doi.org/10.19132/1807-8583201738.197-224.

19. Center for Health Information and Analysis. Betsy Lehman center for patient safety and medical error reduction [Internet]. 2015 [cited 02 jan. 2017]. Available from: http://www.mass.gov/chia/gov/betsylehman.html.

20. Barreto Junior IF. Paradoxos entre regulação da mídia e liberdade de expressão na sociedade da informação. In: Anais do VII Congresso Brasileiro da Sociedade da Informação, 2014, São Paulo, Brasil. [Internet]. 2014. [cited 2018 Dec 31]. Available from: http://www.revistaseletronicas.fmu.br/index.php/CBSI/article/view/527.

21. Aiken LH, Sloane DM, Bruyneel L, Van den Heede K, Sermeus W. Nurses' reports of working conditions and hospital quality of care in 12 countries in Europe. Int J Nurs Stud [Internet]. 2013 [cited 2018 Dec 31];50(2):143-53. Available from:

https://doi.org/10.1016/j.ijnurstu.2012.11.009.

22. Ordem dos Enfermeiros (PT). Notícias. [Internet] 2017. [acesso em 28 jul. 2017]. Disponível em:

http://www.ordemenfermeiros.pt/comunicacao/Paginas/Noticias15.aspx

23. Teodosio S, Padilha MI, Enders BC, Lira A, Breda KL. Análise do conceito de Identidade Profissional do Enfermeiro. In: Anais do 6o Congresso Ibero-Americano em Investigação Qualitativa (CIAIQ2017), 2017, Salamanca, Espanha [Internet]. 2017 [cited 2018 Dec 31]. Available from: https://proceedings.ciaiq.org/index.php/ciaiq2017/article/view/1511. 